\title{
A New Demerit Control Chart for Monitoring the Quality of Multivariate Poisson Processes
}

\author{
By Jeh-Nan Pan* \\ Chung-I Lit \\ Min-Hung Huang ${ }^{\dagger}$
}

This study aims to develop a new demerit control chart suitable for monitoring the quality of a manufacturing process with multiple characteristics subject to multivariate weighted Poisson distribution. Considering the correlation among different quality characteristics and their degrees of influence on the final product, we propose a new statistic for demerit scheme which gives different weights to different quality characteristics. Then, a new demerit control chart for multivariate weighted Poisson distribution (WMP chart) is developed accordingly. Moreover, a simulation study is conducted to evaluate the detecting performances of our proposed WMP chart and multivariate Poisson control chart (MP chart) using the out-of-control average run length (ARL_1). Finally, a numerical example with a two dimensional telecommunication data set is given to demonstrate the usefulness of our proposed WMP chart. Both the simulation results and numerical example show that the detecting ability of our proposed WMP chart outperforms that of the MP chart when a process shift occurs. Hopefully, the results of this research can provide a better alternative for detecting the mean shifts occurred in a multivariate Poisson process.

Keywords: Attribute control chart, Average run length, Multiple Quality Characteristics, Multivariate Poisson control chart, Multivariate weighted Poisson distribution.

\section{Introduction}

The SPC control charts play an important role in monitoring and improving the process quality. Attribute control charts are frequently used in traditional manufacturing industries for detecting and removing assignable causes of various types of defects so that the process stability can be closely monitored. Among them, $\mathrm{C}$ chart and $\mathrm{U}$ chart are the most commonly used control charts when the defects of a product follow Poisson distribution. With the advent of modern technology, different types of defects often appear in industrial products with multiple quality characteristics and those defects are likely to interact with each other. For example, different failures observed during testing processes, and different misprints and errors found on manuscript pages can be classified into more than two categories (Cozzucoli and Marozzi, 2018; Laungrungrog et al., 2014). Thus, the traditional $\mathrm{C}$ and $\mathrm{U}$ charts are no longer able to use for monitoring the quality of this kind of industrial products or processes.

\footnotetext{
*Professor and CSQ Fellow, National Cheng Kung University, Taiwan.

*National Cheng Kung University, Taiwan.

${ }^{\dagger}$ National Cheng Kung University, Taiwan.
} 
Many researchers had developed the attribute control charts for multivariate Poisson distribution (Aslam et al., 2017; Chiu and Kuo, 2007; He et al., 2014). Assuming that a product has $\mathrm{p}$ quality characteristics and its defects follow a multivariate Poisson distribution, Chiu and Kuo (2007) defined a statistic D to be a total number of defects for $\mathrm{p}$ quality characteristics and constructed an attribute control chart for multivariate Poisson distribution (MP chart). But, the degrees of influence for these $\mathrm{p}$ quality characteristics on the final product were not considered in their multivariate Poisson control charts. Actually, in many practical applications, not only different quality characteristics but also different types of defects with the same quality characteristic have different impacts on the final product. For example, the insulation paper manufacturing process at Chang Chun Plastics Corporation in Taiwan has two major quality characteristics, i.e. wrinkles and black spots. According to different sizes of the black spots, there are three types of defects with size greater than $2.0 \mathrm{~mm}$, between $1.0 \sim 2.0 \mathrm{~mm}$ and smaller than $1.0 \mathrm{~mm}$. Since the size of black spot has significant effect on the insulation paper, one needs to assign different weights to different types of defects to reflect their severity. However, a suitable attribute control chart for monitoring the quality of such a product is still lacking. Due to the fact that different quality characteristics or different types of defects may have different impacts on the final product, different weights ought to be assigned to different types of defects according to their degrees of influence on the final product. Thus, it becomes necessary to develop a new demerit control chart suitable for monitoring and controlling the quality of a manufacturing process with multiple quality characteristics subject to multivariate weighted Poisson distribution.

\section{Literature Review}

Control charts dealing with attribute data are called attribute control charts. Basically, there are two types of attributes data: go/no-go type data and counting data. Based on the binomial distribution, $\mathrm{P}$ charts and NP are used to monitor go/no-go type or defective data; based on the Poisson distribution, $\mathrm{C}$ charts and $\mathrm{U}$ charts are used to monitor defect count data. Cozzucoli and Marozzi (2018) stated that an important issue in assessing the defect count problem is to monitor simultaneously the number of nonconformities belonging to each of the $\mathrm{k}$ ordered, distinct and not mutually exclusive defect categories. They mentioned that the appropriate probabilistic model in this context is the multivariate Poisson distribution, see e.g. (Johnson et al., 1997; Karlis, 2003; Krishnamoorthy, 1951). Instead of monitoring the number of nonconformities, a demerit statistic is used to simultaneously monitor the counts for different types of defects. The demerit statistic is a linear combination of the counts of these different types of defects. According to Dodge (1928) and Henley and Kumamoto (1981), various types of defects or hazards can be classified into the following four different categories from customer's perspective: 
Class A defects $\left(\mathrm{X}_{1}\right)$ - Very serious or having catastrophic effects: This type of defect or hazard involves potential safety issues. The product is either completely failed to function or will cause subsequent equipment damage and/or multiple injuries/death to personnel.

Class B defects $\left(\mathrm{X}_{2}\right)$ - Serious or having critical effects: High degree of customer dissatisfaction will be caused by this type of defect or failure.

Class $\mathrm{C}$ defects $\left(\mathrm{X}_{3}\right)$ - Moderately serious or having marginal effects: Customer is made uncomfortable or is annoyed by this type of defect. The product performance will be degraded and the maintenance cost will be increased.

Class D defects $\left(\mathrm{X}_{4}\right)$ - Not serious or effects can be negligible: This type of defect will cause a slight customer annoyance. Customer will probably notice only very minor defects in surface finish, appearance or will probably not be able to detect the variation.

Usually, different weights are given according to the severity of different types of defects. Montgomery (2009) defined the demerit scheme $d_{s}$ as follows:

$d_{s}=w_{1} X_{1}+w_{2} X_{2}+w_{3} X_{3}+w_{4} X_{4}$

The most commonly used weights are $w_{1}=100, w_{2}=50, w_{3}=10$ and $\mathrm{w}_{4}=1$ for the above four different types of defects, i.e. Class A, B, C and D respectively. Montgomery (2009) suggested that the classification of the defects severity and their weights can be determined by the users. Assuming that a Poisson process has $\mathrm{p}$ different classes of defects, Jones et al. (1999) used a demerit rating system to monitor the total rating score for different classes of defects. They proposed a demerit control chart based on the exact distribution of linear combinations of independent Poisson random variables. Assuming that different classes of defects are independent of each other, Chimka and Cabrera Arispe (2006) proposed a new method for finding the exact probability distribution of demerit control chart. Their demerit control chart is assumed that different classes of defects are independent of each other. To relieve the assumption of independence, Chiu and Kuo (2007) constructed a demerit control chart for multivariate Poisson distribution (MP chart). They denoted $X_{j}$ to be the number of defects of jth quality characteristics and let $\mathrm{X}_{\mathrm{j}}$ follows a Poisson distribution with mean $\lambda_{j}+\lambda_{0}, j=1,2, \ldots . p$. Note that $\mathbf{X}=\left(X_{1}, \ldots, X_{p}\right)$ follows a multivariate Poisson distribution and the covariance of $\mathrm{X}_{\mathrm{u}}$ and $\mathrm{X}_{\mathrm{v}}$ is $\lambda_{0}$, where $\mathrm{u} \neq \mathrm{v}$ and $\mathrm{u}, \mathrm{v}=$ $1, \ldots, p$. The new statistic $\mathrm{D}$ for the total number of defects of a product with $\mathrm{p}$ quality characteristics is defined as follows:

$D=X_{1}+X_{2}+\cdots+X_{p}=\sum_{j=1}^{p} X_{j}$ 
Based on Holgate's bivariate Poisson model (1964) and the method proposed by Kemp and Kemp (1965), Chiu and Kuo (2007) developed the probability density function for $\mathrm{p}=2$ and $\mathrm{p}=3$ as

$P(D=d)=\exp \left[-\left(\lambda_{1}+\lambda_{2}-\lambda_{0}\right)\right] \sum_{i=0}^{d / 2} \frac{\left(\lambda_{1}+\lambda_{2}-2 \lambda_{0}\right)^{d-2 i} \lambda_{0}^{i}}{(d-2 i) ! i l}, d=0,1,2, \ldots$

and

$\mathrm{P}(\mathrm{D}=\mathrm{d})=\exp \left[-\left(\lambda_{1}+\lambda_{2}+\lambda_{3}-2 \lambda_{0}\right)\right] \sum_{\mathrm{i}=0}^{\mathrm{d} / 3} \frac{\left(\mathrm{\lambda}_{1}+\lambda_{2}+\lambda_{3}-3 \lambda_{0}\right)^{\mathrm{d}-3 i_{\lambda_{0}}^{\mathrm{i}}}}{(\mathrm{d}-3 \mathrm{i}) \mathrm{il}}, \mathrm{d}=0,1,2, \ldots(2)$

The probability density function for $\mathrm{p}>3$ can be derived by using mathematical induction and it is given by

$P(D=d)=\exp \left\{-\left[\sum_{j=1}^{\mathrm{p}} \lambda_{j}-(\mathrm{p}-1) \lambda_{0}\right]\right\} \sum_{\mathrm{i}=0}^{\mathrm{d} / \mathrm{p}} \frac{\left(\sum_{\mathrm{j}=1}^{\mathrm{p}} \lambda_{\mathrm{j}}-\mathrm{p} \lambda_{0}\right)^{\mathrm{d}-\mathrm{pi}} \lambda_{0}^{\mathrm{i}}}{(\mathrm{d}-\mathrm{pi}) ! \mathrm{i} !}, \mathrm{d}=0,1,2, \ldots$

The control limits with probability of type I error $\alpha$ can also be obtained as below:

$$
\begin{aligned}
& \mathrm{P}(\mathrm{D}>U C L)=\sum_{\mathrm{d}=\mathrm{UCL}}^{\infty} \exp \left\{-\left[\sum_{\mathrm{j}=1}^{\mathrm{p}} \lambda_{\mathrm{j}}-(\mathrm{p}-1) \lambda_{0}\right]\right\} \sum_{\mathrm{i}=0}^{\mathrm{d} / \mathrm{p}} \frac{\left(\sum_{\mathrm{j}=1}^{\mathrm{p}} \lambda_{\mathrm{j}}-\mathrm{p} \lambda_{\mathrm{o}}\right)^{\mathrm{d}-\mathrm{pi}} \lambda_{\mathrm{o}}^{\mathrm{i}}}{(\mathrm{d}-\mathrm{pi}) ! \mathrm{i} !} \leq \frac{\alpha}{2}(4) \\
& \mathrm{P}(\mathrm{D}<L C L)=\sum_{\mathrm{d}=0}^{\mathrm{LCL}} \exp \left\{-\left[\sum_{\mathrm{j}=1}^{\mathrm{p}} \lambda_{\mathrm{j}}-(\mathrm{p}-1) \lambda_{0}\right]\right\} \sum_{\mathrm{i}=0}^{\mathrm{d} / \mathrm{p}} \frac{\left(\sum_{\mathrm{j}=1}^{\mathrm{p}} \lambda_{\mathrm{j}}-\mathrm{p} \lambda_{\mathrm{o}}\right)^{\mathrm{d}-\mathrm{pi}}{ }_{\theta_{0}^{\mathrm{i}}}^{\mathrm{i}}}{(\mathrm{d}-\mathrm{pi}) ! \mathrm{i} !} \leq \frac{\alpha}{2}
\end{aligned}
$$

He et al. (2014) developed a cumulative sum control chart for multivariate Poisson distribution (MP-CUMSUM chart). The MP-CUMSUM chart was constructed based on log-likelihood ratio with in-control process parameters. Aslam et al. (2017) adopted the concept of repetitive sampling and proposed a multivariate Poisson control chart. Based on the probability density function of D proposed by Chiu and Kuo (2007), two pairs of control limits (i.e. the outer control limits and inner control limits) of their control chart were obtained for quick detection of the out-of-control process.

\section{Development of a New Demerit Control Chart for Multivariate Weighted Poisson distribution}

\section{Construction of a Probability Function for Multivariate Poisson distribution}

Assuming that a process has p quality characteristics, the number of defects of $\mathrm{jth}$ quality characteristics is denoted by $\mathrm{X}_{\mathrm{j}}$. Let $\mathrm{X}_{\mathrm{j}}$ follows a Poisson distribution with mean $\lambda_{j}+\lambda_{0}, j=1,2, \ldots$. Kawamura (1979) derived the moment generating function (mgf) of the multivariate Poisson distribution based on the limiting form 
mgf for the multivariate binomial distribution. Although Kawamura (1979) mentioned that a random vector $\mathbf{X}=\left(\mathrm{X}_{1}, \mathrm{X}_{2}, \ldots, \mathrm{X}_{\mathrm{p}}\right)$ with the multivariate Poisson distribution can be decomposed into $\left(\mathrm{X}_{10}+\mathrm{X}_{0}, \mathrm{X}_{20}+\mathrm{X}_{0}, \ldots, \mathrm{X}_{\mathrm{p} 0}+\mathrm{X}_{0}\right)$ where $\mathrm{X}_{10}, \ldots, \mathrm{X}_{\mathrm{p} 0}$ and $\mathrm{X}_{0}$ are mutually independent Poisson distribution, he did not provide the probability mass function for a multivariate Poisson distribution. Thus, we are unable to use his approach to derive the probability mass function of weighted sum for our correlated Poisson random variables since the aim of our research is to develop a new demerit control chart. Consequently, it becomes necessary to derive the probability mass function of weighted sum for correlated Poisson random variables. Based on the bivariate Poisson distribution given by Kawamura (1973), we use the principle of mathematical induction to derive the probability function of multivariate Poisson distribution. Let a random vector $\mathbf{X}=\left(\mathrm{X}_{1}, \mathrm{X}_{2}, \ldots, \mathrm{X}_{\mathrm{p}}\right)$ follows a multivariate Poisson distribution, then it can be written as $\mathbf{X} \sim \operatorname{MP}\left(\lambda_{1}, \lambda_{2}, \ldots, \lambda_{\mathrm{p}} ; \lambda_{0}\right)$, where $\lambda_{0}$ is the covariance of $X_{u}$ and $X_{v}$, and $\mathrm{u} \neq \mathrm{v}$ and $\mathrm{u}, \mathrm{v}=1, \ldots, \mathrm{p}$. And the probability function of a multivariate Poisson distribution is written as

$$
\begin{aligned}
& \mathrm{P}(\mathbf{X}=\mathbf{x})=\mathrm{P}\left(\mathrm{X}_{1}=\mathrm{x}_{1}, \mathrm{X}_{2}=\mathrm{x}_{2}, \ldots, \mathrm{X}_{\mathrm{p}}=\mathrm{x}_{\mathrm{p}}\right) \\
& =\sum_{\delta=0}^{\min \left(x_{1}, x_{2}, \ldots, x_{p}\right)}\left[\prod_{j=1}^{p} \frac{\lambda_{j}^{x_{j}-\delta} \lambda_{0}^{\delta}}{\left(x_{j}-\delta\right) ! \delta !} e^{-\lambda_{j}}\right] e^{-\lambda_{0}}
\end{aligned}
$$

where $\mathbf{x}=\left(\mathrm{x}_{1}, \mathrm{x}_{2}, \ldots, \mathrm{x}_{\mathrm{p}}\right)$ and each $\mathrm{X}_{\mathrm{j}}$ follows a Poisson distribution with mean $\lambda_{\mathrm{j}}+\lambda_{0}, \mathrm{j}=1,2, \ldots, \mathrm{p}$.

\section{The Statistics of Demerit Scheme for Multivariate Weighted Poisson Distribution}

Considering that the correlation among different defect classes and their degrees of influence on the final product, we define a new statistics $D_{w}$ for the total number of weighted defects for p quality characteristics and it is given by:

$D_{w}=w_{1} X_{1}+w_{2} X_{2}+\cdots+w_{p} X_{p}$

where $w_{j}$ is the weight of jth quality characteristic. Based on (Holgate, 1964; Kemp and Kemp, 1965), we can derive the probability density function of $\mathrm{D}_{\mathrm{w}}$ for $\mathrm{p}=2$ as follows:

$$
\begin{aligned}
& P\left(D_{w}=d_{1}\right)=P\left(w_{1} X_{1}+w_{2} X_{2}=d_{1}\right) \\
& =e^{-\left(\lambda_{0}+\lambda_{1}+\lambda_{2}\right)} \sum_{d_{2}=0}^{d_{1}} \sum_{d=0}^{\min \left(\frac{d_{1}-d_{2}}{w_{1}}, \frac{d_{2}}{w_{2}}\right)} \frac{\frac{\lambda_{1}-d_{1}}{w_{1}}-\lambda_{2}^{\frac{d_{2}}{w_{2}}-d} \lambda_{0}^{d}}{\left(\frac{d_{1}-d_{2}}{w_{1}}-d\right) !\left(\frac{d_{2}}{w_{2}}-d\right) ! d !}, d_{1}=0,1,2, \ldots
\end{aligned}
$$


For $\mathrm{p}=3$, the probability density function of $\mathrm{D}_{\mathrm{w}}$ as listed in Eq. (8) can be derived based on the method of variable transformation.

$P\left(D_{w}=d_{1}\right)=P\left(w_{1} X_{1}+w_{2} X_{2}+w_{3} X_{3}=d_{1}\right)$
$=e^{-\sum_{j=0}^{3} \lambda_{1}} \sum_{d_{2}=0}^{d_{1}} \sum_{d_{3}=0}^{d_{2}} \sum_{d=0}^{a_{4}} \frac{\lambda_{1}^{a_{1}} \lambda_{2}^{a_{2}} \lambda_{3}^{a_{3}} \lambda_{0}^{\mathrm{d}}}{\left(a_{1}\right) !\left(a_{2}\right) !\left(a_{3}\right) ! d !}, d_{1}=0,1,2, \ldots$

where $a_{1}=\frac{d_{1}-d_{2}}{w_{1}}-d, a_{2}=\frac{d_{2}-d_{3}}{w_{2}}-d, a_{3}=\frac{d_{3}}{w_{3}}-d, a_{4}=\min \left(\frac{d_{1}-d_{2}}{w_{1}}, \frac{d_{2}}{w_{3}}\right)$.

Following similar derivation shown in Eq. (8), we use the principle of mathematical induction and derive the probability density function of $\mathrm{D}_{w}$ when the number of quality characteristics is larger than 3. A general probability density function of $\mathrm{D}_{\mathrm{w}}$ can be written as follow:

$P\left(D_{w}=d_{1}\right)=P\left(\sum_{j=1}^{p} w_{j} X_{j}=d_{1}\right)$

$=\mathrm{e}^{-\sum_{\mathrm{j}=0}^{\mathrm{p}} \lambda_{\mathrm{j}}} \sum_{\mathrm{d}_{2}=0}^{\mathrm{d}_{1}} \ldots \sum_{\mathrm{d}=0}^{\mathrm{a}_{\mathrm{p}+1}} \frac{\lambda_{0}^{\mathrm{d}}}{\mathrm{d} !}\left[\prod_{\mathrm{j}=1}^{\mathrm{p}} \frac{\lambda_{\mathrm{j}}^{\mathrm{a}_{\mathrm{j}}}}{\mathrm{a}_{\mathrm{j}} !}\right], \mathrm{d}_{1}=0,1,2, \ldots$

where $a_{i}=\frac{d_{i}-d_{i+1}}{w_{1}}-d, i=1, \ldots,(p-1)$ and $a_{p+1}=\min \left(\frac{d_{1}-d_{2}}{w_{1}}, \frac{d_{2}-d_{3}}{w_{2}}, \ldots, \frac{d_{p}}{w_{p}}\right)$.

Note that the probability density function of D proposed by Chiu and Kuo (2007) is a special case of our general probability density function of $D_{w}$ when the weights of $\mathrm{p}$ quality characteristics are equal to 1 . For $\mathrm{w}_{1}=\mathrm{w}_{2}=\cdots=\mathrm{w}_{\mathrm{p}}=1$, it can be shown that the probability density function listed in Eq. (3) is equivalent to the probability density function listed in Eq. (9). Thus, the correctness of the general pdf of $\mathrm{D}_{\mathrm{w}}$ is further confirmed.

\section{Establishing a Multivariate Weighted Poisson Control Chart}

Based on the general probability density function as listed in Eq. (9), the upper control limit (UCL) and lower control limit (LCL) with the probability of type I error $\alpha$ can be obtained by solving the following equations:

$$
\begin{aligned}
& \mathrm{P}\left(\mathrm{D}_{\mathrm{w}}>U C L\right)=\sum_{\mathrm{d}_{\mathrm{i}}=\mathrm{UCL}}^{\infty} \mathrm{P}\left(\mathrm{D}_{\mathrm{w}}=\mathrm{d}_{\mathrm{i}}\right) \leq \frac{\alpha}{2} \\
& \mathrm{P}\left(\mathrm{D}_{\mathrm{w}}<L C L\right)=\sum_{\mathrm{d}_{\mathrm{i}}=0}^{\mathrm{LCL}} \mathrm{P}\left(\mathrm{D}_{\mathrm{w}}=\mathrm{d}_{\mathrm{i}}\right) \leq \frac{\alpha}{2}
\end{aligned}
$$

For Phase II monitoring, the in-control parameters are estimated from the historical data set using the maximum likelihood estimation (MLE) method. Then, the in-control parameters are used to construct the new proposed multivariate weighted Poisson control chart (WMP chart). 


\section{Simulation Analysis}

In the simulation study, we first compare the detecting performance of MP chart proposed by Chiu and Kuo (2007) with that of our proposed WMP chart under different correlation coefficients. Then, a sensitive analysis for WMP charts is performed under different weight combinations of the defects. In statistical process control, the out-of-control ARL $\left(\mathrm{ARL}_{1}=1 /(1-\beta)\right.$, where $\beta$ is the probability of a type II error) is commonly used to evaluate and compare the detecting performance among different control charts when the in-control ARL $\left(\mathrm{ARL}_{0}=1 / \alpha\right.$, where $\alpha$ is the probability of a type I error or a false alarm) is fixed at $1 / 0.0027=370$. The values of $\alpha$ and $1-\beta$ are calculated as $1-P\left\{L C L \leq D_{w} \leq \mathrm{UCL}\right\}$ when the random variable $\mathrm{D}_{w}$ has the probability mass function in Eq.(7) with the in-control and out-of-control parameters, respectively.

\section{The Parameters settings for Multivariate Poisson Control Chart}

The multivariate Poisson process with two quality characteristics is used as an example to explain the results of our simulation studies. Assuming that the two quality characteristics in a process follow a multivariate Poisson distribution

$\mathbf{X}=\left(\mathrm{X}_{1}, \mathrm{X}_{2}\right) \sim \operatorname{MP}\left(\lambda_{1}, \lambda_{2} ; \lambda_{0}\right)$,

where $\lambda_{0}$ is the covariance of $X_{1}$ and $X_{2}$ (i.e. it is the average number of defects occurred in both quality characteristics), $\lambda_{1}$ and $\lambda_{2}$ are the number of mean defects that only appear in quality characteristic $\mathrm{X}_{1}$ and $\mathrm{X}_{2}$, respectively.

In performing the simulation analysis, we often fix the type I error $\alpha$ at 0.0027 (i.e. the ARL0 is fixed at 370). However, due to the fact that defect data are discrete variables, a unit change of the control limits for attribute control charts will result in a significant difference for their corresponding probabilities of type I error. Thus, we need to adjust the upper control limits so that their corresponding probabilities of type I error $\alpha$ are closer to 0.0027 . For example, if LCL $=2$ and $\mathrm{UCL}=22$, then its corresponding $\alpha=0.00178$; When $\mathrm{LCL}=2$ and $\mathrm{UCL}=21$, then its corresponding $\alpha=0.00296$. As the probability type I error $\alpha=0.00296$ is close to 0.0027 , we select $\mathrm{UCL}=21$ as the upper control limit for the multivariate Poisson control chart. To compare the detecting performances of our proposed WMP control charts with that of MP chart (with weight $\mathrm{w}_{1}=1$ and $\mathrm{w}_{2}=1$ ), the simulation settings for bivariate Poisson control charts under various weight combinations are listed in Table 1.

Table 1. List of Common Weights for Bivariate Weighted Poisson Control Chart

\begin{tabular}{|l|c|c|c|c|c|c|c|c|c|c|c|}
\hline & \multicolumn{10}{|c|}{ Bivariate Poisson control charts } \\
\hline & MP chart & & \multicolumn{10}{|c|}{ WMP chart } \\
\hline$w_{1}$ & 1 & & 2 & 3 & 4 & 5 & 10 & 15 & 20 & 50 & 100 \\
\hline$w_{2}$ & 1 & & 1 & 1 & 1 & 1 & 1 & 1 & 1 & 1 & 1 \\
\hline
\end{tabular}


By fixing $\mathrm{ARL}_{0}$ at 370 approximately, various upper and lower control limits of bivariate Poisson control charts under the different combinations of weights and correlations can be obtained by letting the probability type I error $\alpha$ be close to 0.0027. For example, given weight $\left(\mathrm{w}_{1}, \mathrm{w}_{2}\right)=(1,1)$ and correlation coefficient $\rho=0.75$, the $L C L=55$ and $U C L=130$ for MP chart; while given $\left(\mathrm{w}_{1}, \mathrm{w}_{2}\right)=(2,1)$ and correlation coefficient $\rho=0.75$, the $\mathrm{LCL}=90$ and $\mathrm{UCL}=207$ for WMP chart. Similarly, the other pairs of LCL/UCL for the rest combinations of weights and correlation coefficients are summarized in Table 2.

Table 2. Control Limits under Different Combinations of Weights and Correlations

\begin{tabular}{|c|c|c|c|c|c|c|c|c|c|c|}
\hline & & \multicolumn{10}{|c|}{$\left(w_{1}, w_{2}\right)$} \\
\hline $\begin{array}{c}\text { LCL } \\
\text { UCL }\end{array}$ & $(1,1)$ & $(2,1)$ & $(3,1)$ & $(4,1)$ & $(5,1)$ & $(10,1)$ & $(15,1)$ & $(20,1)$ & $(50,1)$ & $(100,1)$ \\
\hline \multirow{2}{*}{$\rho=0.75$} & 55 & 90 & 124 & 158 & 186 & 357 & 523 & 687 & 1677 & 3328 \\
\cline { 2 - 13 } & 130 & 207 & 284 & 362 & 434 & 824 & 1211 & 1594 & 3904 & 7756 \\
\hline \multirow{2}{*}{$\rho=0.50$} & 24 & 38 & 55 & 71 & 87 & 167 & 237 & 312 & 762 & 1513 \\
\cline { 2 - 12 } & 72 & 117 & 166 & 215 & 264 & 510 & 744 & 984 & 2424 & 4826 \\
\hline \multirow{2}{*}{$\rho=0.25$} & 15 & 23 & 33 & 46 & 57 & 106 & 158 & 208 & 506 & 1008 \\
\cline { 2 - 12 } & 51 & 86 & 124 & 165 & 205 & 398 & 596 & 791 & 1958 & 3911 \\
\hline
\end{tabular}

Since $\mathrm{ARL}_{1}$ is the average number of samples that must be taken to detect a parameter shift when the process is out of control, we consider the following two scenarios for the parameter shifts:

i. There is a shift in either parameter $\lambda_{1}$ or $\lambda_{2}$ (i.e. the parameter $\lambda_{1}$ is changed to $\delta \lambda_{1}$ or the parameter $\lambda_{2}$ is changed to $\delta \lambda_{2}$ ). Thus, the first scenario can be denoted as $\lambda_{1}^{\prime}=\delta \lambda_{1}$ or $\lambda_{2}^{\prime}=\delta \lambda_{2}$

ii. There are simultaneous shifts in both parameters $\lambda_{1}$ and $\lambda_{2}$ (i.e. the parameter $\lambda_{1}$ is changed to $\delta \lambda_{1}$ and the parameter $\lambda_{2}$ is changed to $\delta \lambda_{2}$ ). Thus, the second scenario can be denoted as $\lambda_{1}^{\prime}=\delta \lambda_{1}$ and $\lambda_{2}^{\prime}=\delta \lambda_{2}$

When $\delta=1$, then $\lambda_{1}^{\prime}=\lambda_{1}$ and $\lambda_{2}^{\prime}=\lambda_{2}$. This situation denotes that the process is in statistical control and the out-of-control average run length is equal to $\mathrm{ARL}_{0}$. When the magnitudes of scale shift $(\delta)$ are set as $0.5,0.8,1,1.3$ and 1.5, then the process is out-of-control and its $\mathrm{ARL}$ is equal to $\mathrm{ARL}_{1}$.

\section{Simulation Results}

Without loss of generality, the parameters $\lambda_{1}$ and $\lambda_{2}$ are set as 20 and 4, respectively. In the simulation studies, we compare the detecting performances of MP charts with various parameter shifts to those of WMP charts under high correlation $(\rho=0.75)$, medium correlation $(\rho=0.5)$ and low correlation 
$(\rho=0.25)$. Under various combinations of correlation coefficients $\rho$ and weights, the out-of-control ARLs for detecting shift in either parameter $\lambda_{1}$ or $\lambda_{2}$ are listed in Table 3 and 4 respectively.

Table 3. The ARLs of MP and WMP Charts for Detecting Parameter Shifts in $\lambda_{-} 1$ under Various Combinations of $\rho$ and Weights

\begin{tabular}{|l|c|c|c|c|c|c|c|c|c|c|c|}
\hline & & \multicolumn{7}{|c|}{$\left(\mathrm{W}_{1}, \mathrm{~W}_{2}\right)$} \\
\hline$\rho$ & $\delta$ & $(1,1)$ & $(2,1)$ & $(3,1)$ & $(4,1)$ & $(5,1)$ & $(10,1)$ & $(15,1)$ & $(20,1)$ & $(50,1)$ & $(100,1)$ \\
\hline 0.75 & 0.5 & 106.1 & 45.7 & 34.3 & 31.4 & 38.5 & 24.8 & 23.8 & 24.7 & 24.7 & 23.8 \\
\hline & 0.8 & 352.7 & 224.3 & 190.3 & 187.8 & 248.9 & 162.5 & 155.1 & 162.6 & 162.6 & 155.1 \\
\hline & $\mathbf{1 . 0}$ & $\mathbf{3 7 8 . 3}$ & $\mathbf{3 7 2 . 0}$ & $\mathbf{3 6 5 . 2}$ & $\mathbf{3 7 5 . 0}$ & $\mathbf{3 6 9 . 8}$ & $\mathbf{3 6 9 . 4}$ & $\mathbf{3 7 1 . 6}$ & $\mathbf{3 6 8 . 4}$ & $\mathbf{3 6 8 . 4}$ & $\mathbf{3 7 1 . 2}$ \\
\hline & 1.3 & 130.5 & 105.8 & 93.0 & 85.8 & 65.6 & 73.2 & 71.5 & 69.0 & 69.0 & 70.0 \\
\hline & 1.5 & 59.6 & 39.9 & 32.5 & 28.9 & 22.3 & 23.0 & 21.9 & 20.9 & 20.8 & 20.9 \\
\hline 0.50 & 0.5 & 18.6 & 18.1 & 11.7 & 10.2 & 9.3 & 7.7 & 9.3 & 9.3 & 9.3 & 8.8 \\
\hline & 0.8 & 122.0 & 180.0 & 116.7 & 104.4 & 96.8 & 85.4 & 103.3 & 103.3 & 103.3 & 98.0 \\
\hline & $\mathbf{1 . 0}$ & $\mathbf{3 6 5 . 1}$ & $\mathbf{3 7 7 . 4}$ & $\mathbf{3 6 5 . 7}$ & $\mathbf{3 6 9 . 6}$ & $\mathbf{3 6 7 . 8}$ & $\mathbf{3 7 1 . 4}$ & $\mathbf{3 6 9 . 6}$ & $\mathbf{3 6 9 . 6}$ & $\mathbf{3 6 9 . 6}$ & $\mathbf{3 7 1 . 0}$ \\
\hline & 1.3 & 115.0 & 45.5 & 45.7 & 45.4 & 45.1 & 45.8 & 35.1 & 35.1 & 35.1 & 35.5 \\
\hline & 1.5 & 36.6 & 13.6 & 12.8 & 12.3 & 12.0 & 11.6 & 9.3 & 9.2 & 9.2 & 9.3 \\
\hline 0.25 & 0.5 & 8.3 & 11.4 & 9.0 & 5.7 & 5.2 & 5.6 & 4.9 & 4.9 & 5.6 & 4.9 \\
\hline & 0.8 & 84.6 & 201.3 & 64.8 & 83.6 & 74.0 & 83.8 & 69.4 & 69.4 & 83.8 & 69.4 \\
\hline & $\mathbf{1 . 0}$ & $\mathbf{3 9 1 . 4}$ & $\mathbf{3 6 9 . 4}$ & $\mathbf{3 6 7 . 7}$ & $\mathbf{3 6 9 . 1}$ & $\mathbf{3 6 9 . 8}$ & $\mathbf{3 7 0 . 2}$ & $\mathbf{3 7 0 . 4}$ & $\mathbf{3 7 0 . 4}$ & $\mathbf{3 7 0 . 6}$ & $\mathbf{3 7 0 . 4}$ \\
\hline & 1.3 & 66.7 & 23.1 & 21.5 & 26.6 & 28.2 & 24.7 & 28.0 & 28.0 & 24.8 & 28.0 \\
\hline & 1.5 & 17.0 & 6.6 & 6.0 & 6.9 & 7.1 & 6.5 & 7.0 & 7.0 & 6.5 & 7.0 \\
\hline
\end{tabular}


Vol. 5, No. $4 \quad$ Pan et al.: A New Demerit Control Chart for Monitoring the Quality...

Table 4. The ARLs of MP and WMP Charts for Detecting Parameter Shift in $\lambda \_2$ under Various Combinations of $\rho$ and Weights

\begin{tabular}{|c|c|c|c|c|c|c|c|c|c|c|c|}
\hline & \multicolumn{7}{|c|}{$\left(\mathrm{W}_{1}, \mathrm{~W}_{2}\right)$} \\
\hline$\rho$ & $\delta$ & $(1,1)$ & $(2,1)$ & $(3,1)$ & $(4,1)$ & $(5,1)$ & $(10,1)$ & $(15,1)$ & $(20,1)$ & $(50,1)$ & $(100,1)$ \\
\hline 0.75 & 0.5 & 412.9 & 361.9 & 353.3 & 356.4 & 381.1 & 366.0 & 368.0 & 364.0 & 364.0 & 367.1 \\
\hline & 0.8 & 403.2 & 372.5 & 362.8 & 366.4 & 375.2 & 367.7 & 369.7 & 366.2 & 366.2 & 369.1 \\
\hline & $\mathbf{1 . 0}$ & $\mathbf{3 7 8 . 3}$ & $\mathbf{3 7 2 . 0}$ & $\mathbf{3 6 5 . 2}$ & $\mathbf{3 7 0 . 8}$ & $\mathbf{3 6 9 . 8}$ & $\mathbf{3 6 9 . 4}$ & $\mathbf{3 7 1 . 6}$ & $\mathbf{3 6 8 . 4}$ & $\mathbf{3 6 8 . 4}$ & $\mathbf{3 7 1 . 2}$ \\
\hline & 1.3 & 324.7 & 360.2 & 362.9 & 373.5 & 359.6 & 372.1 & 375.3 & 372.0 & 372.0 & 375.1 \\
\hline & 1.5 & 285.1 & 346.1 & 357.4 & 372.7 & 351.6 & 373.6 & 377.9 & 374.4 & 374.4 & 377.8 \\
\hline 0.50 & 0.5 & 234.2 & 384.5 & 337.2 & 340.1 & 339.7 & 344.7 & 364.6 & 364.4 & 364.4 & 366.0 \\
\hline & 0.8 & 320.1 & 390.6 & 358.8 & 360.5 & 358.5 & 363.7 & 366.7 & 366.6 & 366.6 & 368.1 \\
\hline & $\mathbf{1 . 0}$ & $\mathbf{3 6 5 . 1}$ & $\mathbf{3 7 7 . 4}$ & $\mathbf{3 6 5 . 7}$ & $\mathbf{3 6 9 . 6}$ & $\mathbf{3 6 7 . 8}$ & $\mathbf{3 7 1 . 4}$ & $\mathbf{3 6 9 . 6}$ & $\mathbf{3 6 9 . 6}$ & $\mathbf{3 6 9 . 6}$ & $\mathbf{3 7 1 . 0}$ \\
\hline & 1.3 & 376.8 & 338.1 & 363.3 & 375.7 & 376.7 & 377.3 & 375.2 & 375.1 & 375.1 & 377.3 \\
\hline & 1.5 & 343.6 & 304.8 & 353.9 & 374.5 & 379.2 & 379.0 & 378.7 & 378.7 & 378.7 & 382.1 \\
\hline 0.25 & 0.5 & 204.5 & 447.0 & 409.4 & 342.8 & 343.6 & 354.4 & 366.6 & 366.6 & 354.5 & 366.6 \\
\hline & 0.8 & 322.8 & 413.4 & 391.0 & 362.6 & 361.6 & 362.0 & 367.2 & 367.2 & 362.1 & 367.2 \\
\hline & $\mathbf{1 . 0}$ & $\mathbf{3 9 1 . 4}$ & $\mathbf{3 6 9 . 4}$ & $\mathbf{3 6 7 . 7}$ & $\mathbf{3 6 9 . 1}$ & $\mathbf{3 6 9 . 8}$ & $\mathbf{3 7 0 . 2}$ & $\mathbf{3 7 0 . 4}$ & $\mathbf{3 7 0 . 4}$ & $\mathbf{3 7 0 . 6}$ & $\mathbf{3 7 0 . 4}$ \\
\hline & 1.3 & 392.4 & 293.8 & 323.5 & 368.6 & 377.4 & 382.4 & 379.1 & 379.1 & 384.0 & 379.1 \\
\hline & 1.5 & 325.5 & 246.3 & 291.8 & 361.2 & 378.6 & 388.2 & 386.0 & 386.0 & 391.7 & 386.6 \\
\hline
\end{tabular}

Moreover, the out-of-control ARLs for detecting the simultaneous shifts in both parameters $\lambda_{1}$ and $\lambda_{2}$ under various combinations of correlation coefficients $\rho$ and weights are listed in Table 5. Notice that a unit change of the control limits for attribute control charts will result in a significant difference for their corresponding probabilities of type I error. Thus, we need to adjust the upper control limits for in-control ARLs in Tables 3,4 and 5 so that their associated probabilities of type I error $\alpha$ are closer to 0.0027 . This is why all the in-control ARLs $\left(\mathrm{ARL}_{0}\right)$ in these Tables are approximately closed to 370 when the scale parameter $(\delta)$ are set to one (1). It is also worthy to note that the out-of-control ARLs in Table 3 are more sensitive to the parameter shifts than those listed in Table 4. This result is expected since we assume that the importance of quality characteristic $\mathrm{X}_{1}$ is greater than quality characteristic $\mathrm{X}_{2}$ (i.e. the heavier weights are assigned to quality characteristic $\mathrm{X}_{1}$ ) in this simulation study. 
Table 5. The ARLs of MP and WMP Charts for Detecting Simultaneous Parameter Shifts in Both $\lambda \_1$ and $\lambda \_2$ under Various Combinations of $\rho$ and Weights

\begin{tabular}{|l|c|c|c|c|c|c|c|c|c|c|c|}
\hline & & \multicolumn{7}{|c|}{$\left(\mathrm{W}_{1}, \mathrm{~W}_{2}\right)$} \\
\hline$\rho$ & $\delta$ & $(1,1)$ & $(2,1)$ & $(3,1)$ & $(4,1)$ & $(5,1)$ & $(10,1)$ & $(15,1)$ & $(20,1)$ & $(50,1)$ & $(100,1)$ \\
\hline 0.75 & 0.5 & 68.8 & 35.1 & 28.4 & 25.1 & 33.8 & 23.0 & 21.9 & 23.0 & 23.0 & 21.9 \\
\hline & 0.8 & 312.8 & 200.9 & 174.8 & 160.1 & 235.4 & 156.0 & 149.4 & 156.2 & 156.2 & 149.4 \\
\hline & $\mathbf{1 . 0}$ & $\mathbf{3 7 8 . 3}$ & $\mathbf{3 7 2 . 0}$ & $\mathbf{3 6 5 . 2}$ & $\mathbf{3 7 5 . 0}$ & $\mathbf{3 6 9 . 8}$ & $\mathbf{3 6 9 . 4}$ & $\mathbf{3 7 1 . 6}$ & $\mathbf{3 6 8 . 4}$ & $\mathbf{3 6 8 . 4}$ & $\mathbf{3 7 1 . 2}$ \\
\hline & 1.3 & 102.5 & 91.4 & 83.9 & 84.5 & 61.5 & 70.8 & 70.3 & 67.9 & 67.9 & 69.3 \\
\hline & 1.5 & 41.4 & 32.2 & 28.0 & 27.2 & 20.4 & 21.9 & 21.3 & 20.6 & 20.6 & 20.8 \\
\hline 0.50 & 0.5 & 11.0 & 12.8 & 9.2 & 8.5 & 8.0 & 7.5 & 8.3 & 8.3 & 8.3 & 8.0 \\
\hline & 0.8 & 93.1 & 150.2 & 102.4 & 94.2 & 89.1 & 82.4 & 98.2 & 98.2 & 98.2 & 94.5 \\
\hline & $\mathbf{1 . 0}$ & $\mathbf{3 6 5 . 5}$ & $\mathbf{3 4 9 . 1}$ & $\mathbf{3 7 6 . 2}$ & $\mathbf{3 6 2 . 7}$ & $\mathbf{3 5 2 . 4}$ & $\mathbf{3 5 9 . 3}$ & $\mathbf{3 6 0 . 7}$ & $\mathbf{3 6 1 . 2}$ & $\mathbf{3 5 8 . 5}$ & $\mathbf{3 7 1 . 0}$ \\
\hline & 1.3 & 80.1 & 37.6 & 40.1 & 41.1 & 41.6 & 43.8 & 34.5 & 34.5 & 34.5 & 35.2 \\
\hline & 1.5 & 22.2 & 10.7 & 10.8 & 10.8 & 10.8 & 11.0 & 9.1 & 9.1 & 9.1 & 9.2 \\
\hline 0.25 & 0.5 & 4.6 & 7.4 & 6.8 & 4.7 & 4.5 & 4.8 & 4.4 & 4.4 & 4.8 & 4.4 \\
\hline & 0.8 & 59.7 & 158.0 & 136.6 & 73.8 & 67.3 & 76.2 & 65.8 & 65.8 & 76.2 & 65.8 \\
\hline & $\mathbf{1 . 0}$ & $\mathbf{3 9 1 . 4}$ & $\mathbf{3 6 9 . 4}$ & $\mathbf{3 6 7 . 7}$ & $\mathbf{3 6 9 . 1}$ & $\mathbf{3 6 9 . 8}$ & $\mathbf{3 7 0 . 2}$ & $\mathbf{3 7 0 . 4}$ & $\mathbf{3 7 0 . 4}$ & $\mathbf{3 7 0 . 6}$ & $\mathbf{3 7 0 . 4}$ \\
\hline & 1.3 & 42.5 & 18.8 & 18.7 & 23.8 & 25.8 & 23.3 & 26.8 & 26.8 & 23.4 & 26.8 \\
\hline & 1.5 & 9.8 & 5.2 & 5.2 & 6.1 & 6.5 & 6.0 & 6.6 & 6.6 & 6.0 & 6.6 \\
\hline
\end{tabular}

It is worthy to note that the performance of WMP is worse than MP chart when the process parameter shifts occurred in the single quality characteristic with a minor impact on the final product. This result is expected since the proposed WMP chart is designed when the impact of each quality characteristic on the final product is different, i.e. our proposed WMP control chart is expected to have better performance for detecting the parameters shifts in the quality characteristic with a serious impact on the final product.

Moreover, the out-of-control ARLs in Table 3 are shorter when the correlation coefficient $\rho$ is smaller and the magnitude of scale shift $(\delta)$ is larger, especially when $\delta=0.5$ and 1.5 . Similar results can be found in Table 5 when simultaneous shifts occurred in both parameters $\lambda_{1}$ and $\lambda_{2}$.

Based on the above simulation results, one can conclude that when the situations under parameter shifts in $\lambda_{1}$ and simultaneous shifts occurred in both 
parameters $\lambda_{1}$ and $\lambda_{2}$ for different correlation coefficients and various shifts in scale parameter (either $\delta>1$ or $\delta<1$ ) for the two quality characteristics, the out-ofcontrol ARLs of our proposed WMP charts are significantly smaller than those of the MP charts, which means that our proposed WMP charts will be able to detect the parameter shifts earlier than the MP charts under these two situations.

\section{Numerical Example}

In this research, a two dimensional telecommunication data set mentioned in Jiang et al. (2002) is used as an example to demonstrate the application of the WMP charts for monitoring a bivariate Poisson process. There are two quality characteristics $\left(\mathrm{X}_{1}\right.$ and $\left.\mathrm{X}_{2}\right)$ and 52 samples in this data set. The procedure for constructing a new control chart for multivariate weighted Poisson distribution is shown as in Figure 1. Based on the preliminary hazards analysis provided by Henley and Kumamoto (1981), one may consider to assign the weights from $\mathrm{w}_{1}=50 \sim 100, \mathrm{w}_{2}=10 \sim 50, \mathrm{w}_{3}=3,4,5$ and $\mathrm{w}_{4}=1,2$ for the four different types of defects or hazards, i.e. Class A, B, C and D respectively according to their severity.

Figure 1. The Procedure for constructing a New Control Chart for Multivariate weighted Poisson distribution

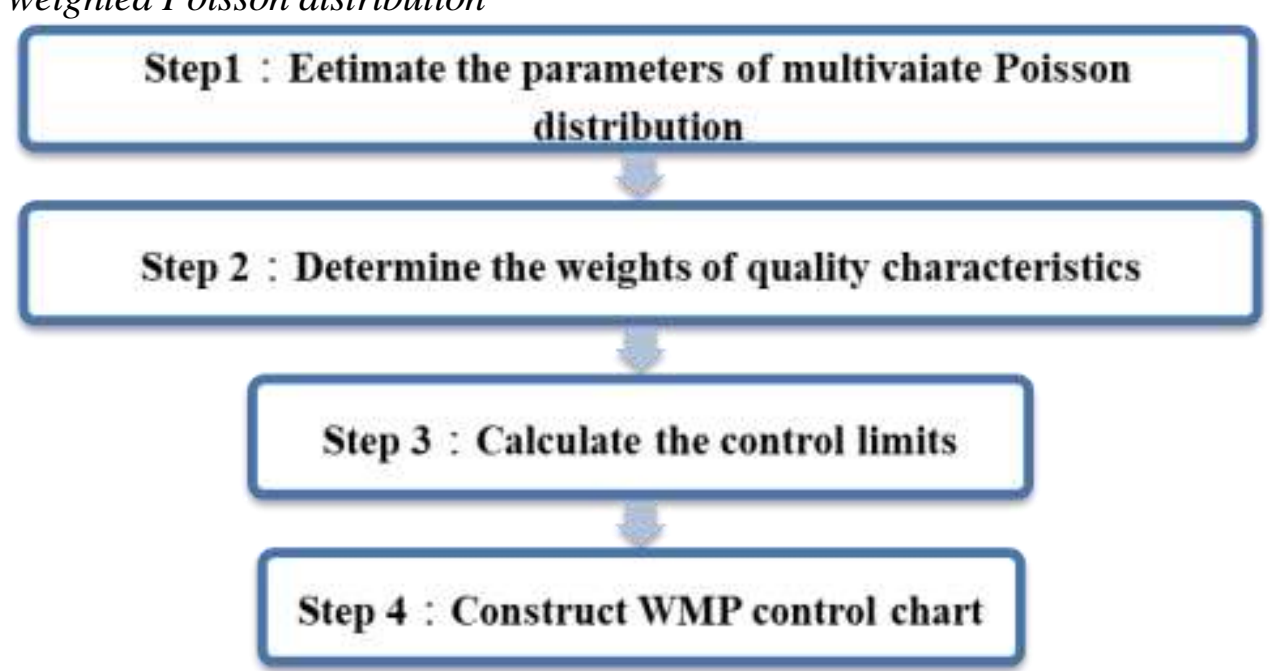

Determination of Process Parameters for the Data Set

In this process, there are two quality characteristics $\mathrm{X}_{1}$ and $\mathrm{X}_{2}$ with means $\lambda_{1}+\lambda_{0}$ and $\lambda_{2}+\lambda_{0}$, respectively. Based on the results shown in Chiu and Kuo (2007) and Jiang et al.(2002), the telecommunication data set follows a bivariate Poisson distribution with mean values of $\mu_{1}=15.31, \mu_{2}=2.35$, and the correlation coefficient $\rho=0.28$. Due to the fact that $\rho=\lambda_{0} / \sqrt{\mu_{1} \mu_{2}}$, the parameter $\lambda_{0}$ can calculated as $\rho \sqrt{\mu_{1} \mu_{2}}=1.68$. The in-control parameters are set 
as $\lambda_{1}=15.31-1.68=13.93, \lambda_{2}=2.35-1.68=0.97$ and $\lambda_{0}=1.68$. Thus, quality characteristics vector $\mathbf{X}$ can be written as:

$\mathbf{X}=\left(\mathrm{X}_{1}, \mathrm{X}_{2}\right) \sim \mathrm{MP}\left(\lambda_{1}=13.93, \lambda_{2}=0.97 ; \lambda_{0}=1.68\right)$

Because the original data set did not consider the weights for these two quality characteristics, we assume that the weights of these two quality characteristics $\mathrm{X}_{1}$ and $\mathrm{X}_{2}$ equal $\mathrm{W}_{1}=10$ and $\mathrm{W}_{2}=1$ according to their degrees of impact to the final results. The in-control parameters are assumed to be known in previous example with telecommunication data set. But, the true in-control parameters may not be available in practical applications. If the in-control parameters are not given, then they need to be estimated from the historical data set. Kawamura (1984) proposed a numerical method for calculating the maximum likelihood estimates $\hat{\lambda}_{1}, \hat{\lambda}_{2}, \hat{\lambda}_{0}$ that are satisfying the following relations and maximizing the logarithm of likelihood function of the bivariate Poisson distribution.

$\hat{\lambda}_{1}+\hat{\lambda}_{0}=\overline{\mathrm{X}}_{1}, \hat{\lambda}_{2}+\hat{\lambda}_{0}=\overline{\mathrm{X}}_{2}, 0 \leq \hat{\lambda}_{0} \leq \min \left(\overline{\mathrm{X}}_{1}, \overline{\mathrm{X}}_{2}\right)$

Moreover, the function simple.bp in the R-package bivpois can be used to calculate the maximum likelihood estimators for bivariate Poisson distribution. See Karlis (2003) for details.

\section{The Construction of WMP Control Chart}

According to Chiu and Kuo (2007), the control limits of the MP control chart for this telecommunication data set are given by:

$$
\left\{\begin{array}{l}
\text { Upper control limit(UCL) }=34 \\
\text { Center line }(\mathrm{CL})=18 \\
\text { Lower control limit }(\mathrm{LCL})=5
\end{array}\right.
$$

Note that the probability of type I error $\alpha$ of the control chart with the MP control limits is equal to 0.00176 . Because the multivariate Poisson control chart has the discrete control limits, it is difficult to determine the control limits with the same level of type I error rate. To fairly compare the detecting performances between two different Poisson control charts, the control limits of our proposed WMP control chart can be determined by setting a closer type I error rate. Thus, the corresponding type I error rate of our proposed WMP chart is set to 0.00182 and its control limits are obtained as follows: 


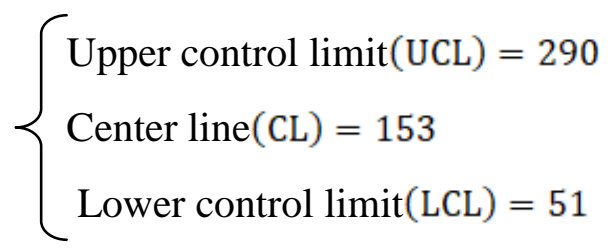

Figure 2 shows that both the MP and WMP control charts are well in statistical control for the 52 telecommunication data set provided by Jiang et al. (2002) since all points are within the control limits. For illustration purpose, we generate another 15 samples with different scales of parameter shifts occurred in parameters $\lambda_{1}$ and $\lambda_{2}$ and add them to the original 52 in-control samples. Then, both MP chart and WMP chart are employed to monitor the multivariate Poisson processes and compare their detecting performances.

Figure 2. WMP Chart vs. MP Chart using the Telecommunication Data Set Provided by Jiang et al. (2002)
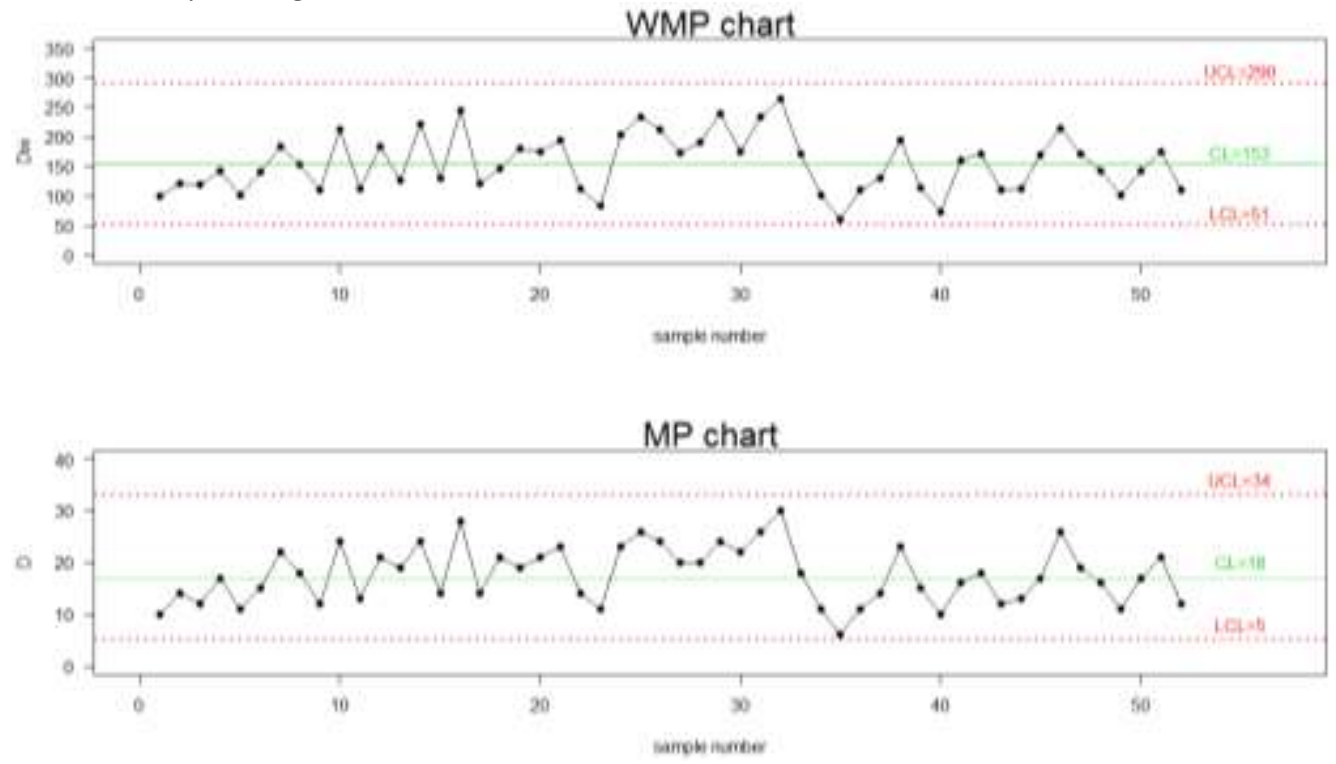

The Process Parameter Shifts Occurred in One Quality Characteristic

When different magnitudes of scale shift occurred in $\lambda_{1}$, the MP and WMP charts are illustrated in Figures 3 and 4. As one can see from Figures 3 and 4, the MP chart did not trigger any out-of-control signal. In contract, the WMP chart triggers an out of control signal at the $54^{\text {th }}$ sample when the magnitude of scale shift for the first parameter equals 0.5 as shown in Figure 3. Another out-ofcontrol signal at $55^{\text {th }}$ samples is triggered by WMP chart when the magnitude of scale shift for the first parameter equals 1.5 as shown in Figure 4. 
Figure 3. WMP Chart vs. MP Chart when the Magnitude of Scale Shift for $X_{1}$ Equals 0.5
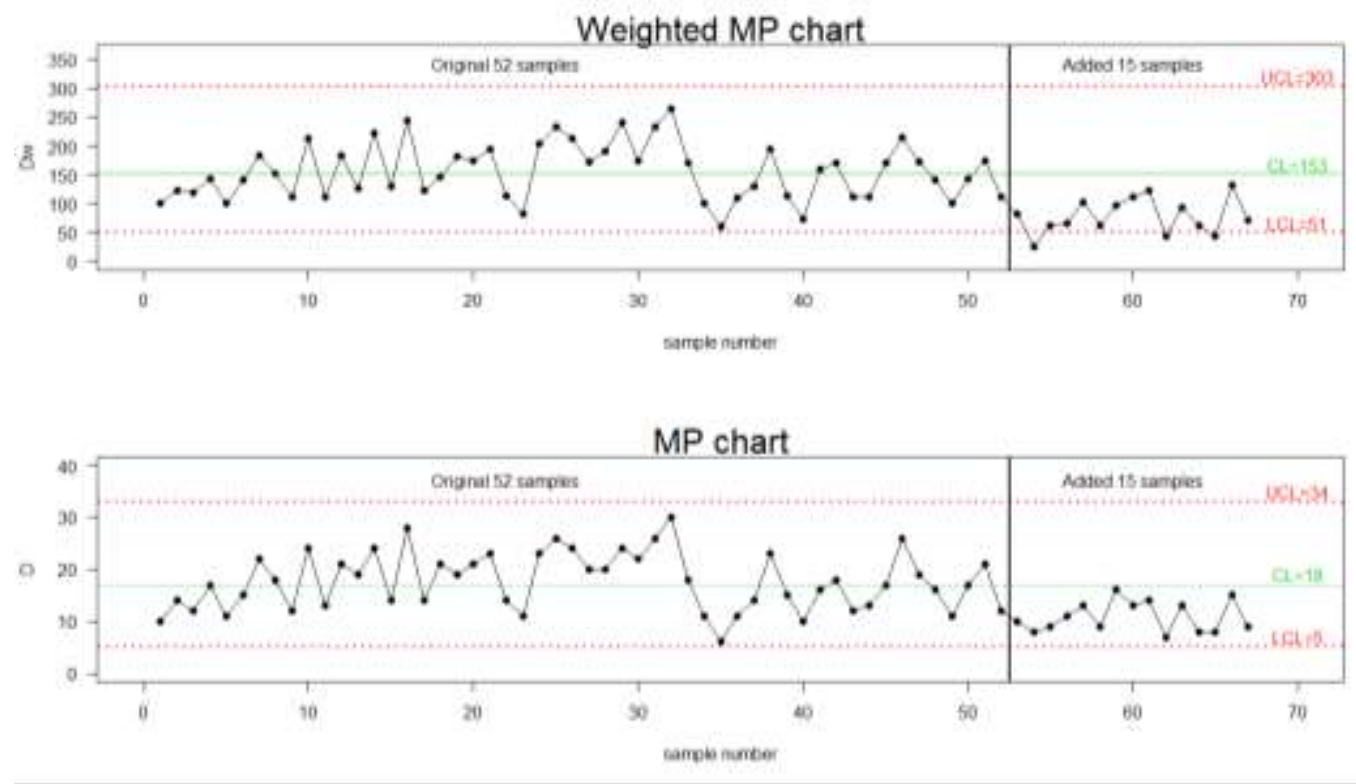

Figure 4. WMP Chart vs. MP Chart when the Magnitude of Scale Shift for $X_{1}$ Equals 1.5
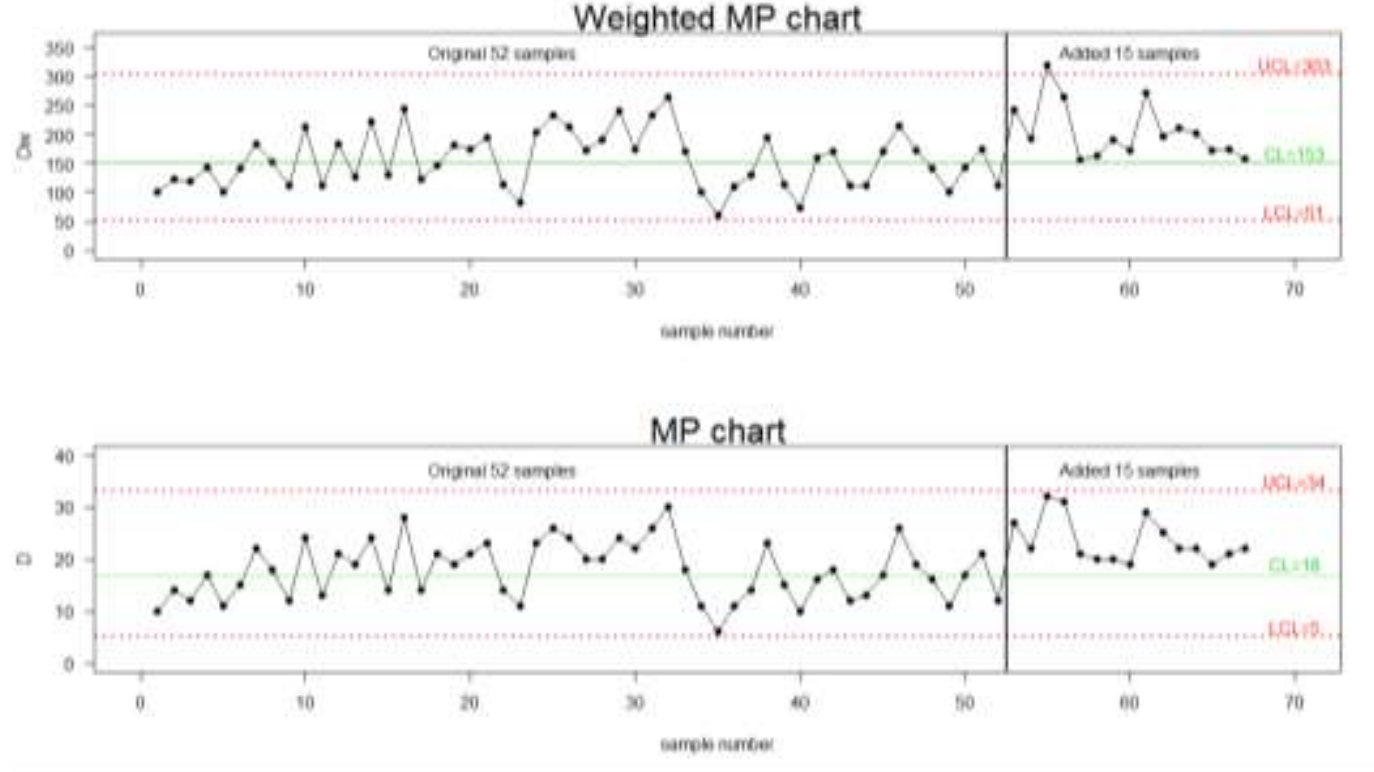

The Process Parameter Shifts Occurred in Both Quality Characteristics

When the simultaneous scale shifts occurred in both parameter $\lambda_{1}$ and $\lambda_{2}$, the MP and WMP charts are illustrated in Figures 5 and 6. As one can see from Figures 5 and 6, the MP chart did not trigger any out-of-control signal. In contract, the WMP chart triggers an out-of-control signal at the $57^{\text {th }}$ sample when the magnitudes of scale shifts for both parameters equal 0.5 as shown in Figure 5. 
Vol. 5, No. $4 \quad$ Pan et al.: A New Demerit Control Chart for Monitoring the Quality...

Another out-of-control signal at $59^{\text {th }}$ sample is triggered by WMP chart when the magnitudes of scale shift for both parameters equal 1.5 as shown in Figures 6 .

Based on the above numerical results, one can conclude that the detecting abilities of our proposed WMP charts outperform those of the MP charts when the parameter has a shift in the quality characteristic with a serious impact on the final product.

Figure 5. WMP Chart vs. MP Chart when the Magnitudes of Scale Shift for both $X_{1}$ and $X_{2}$ Equal 0.5
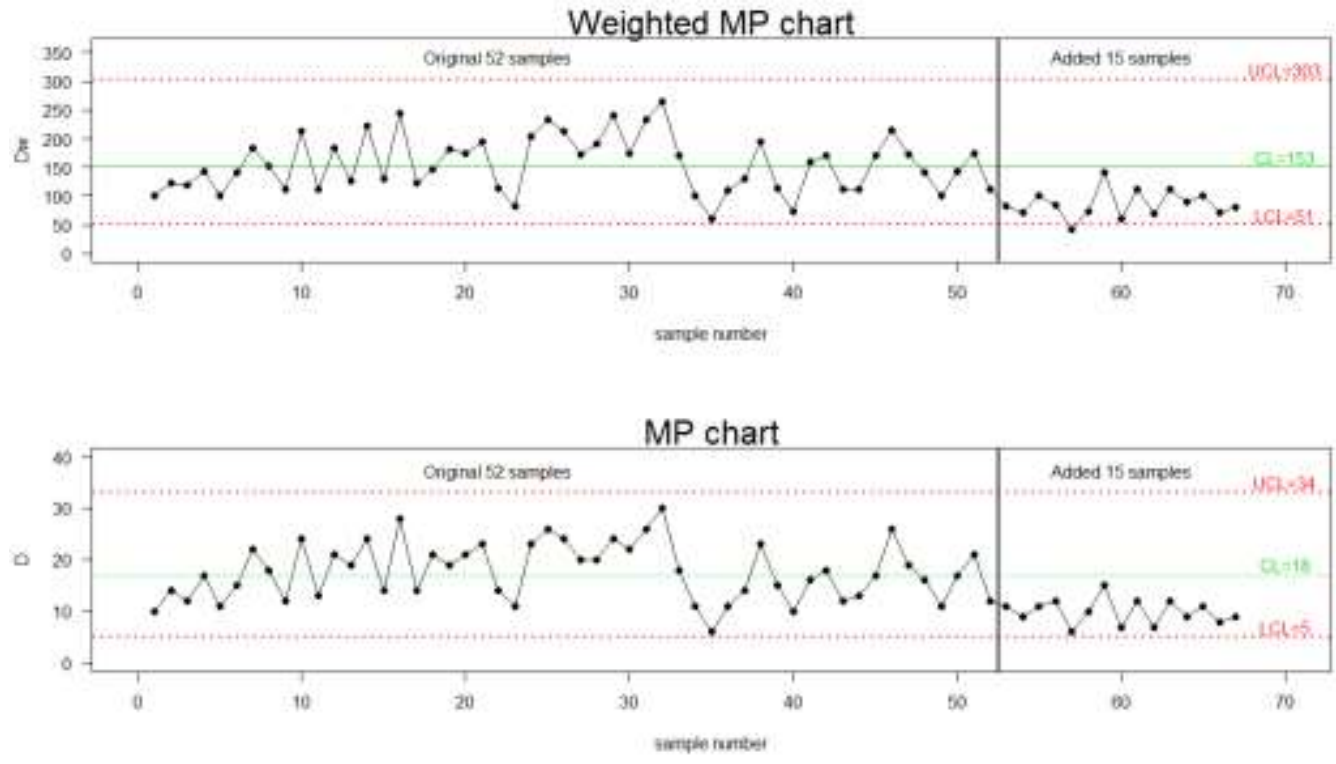

Figure 6. WMP Chart vs. MP Chart when the Magnitudes of Scale Shift for both $X_{1}$ and $X_{2}$ Equal 1.5
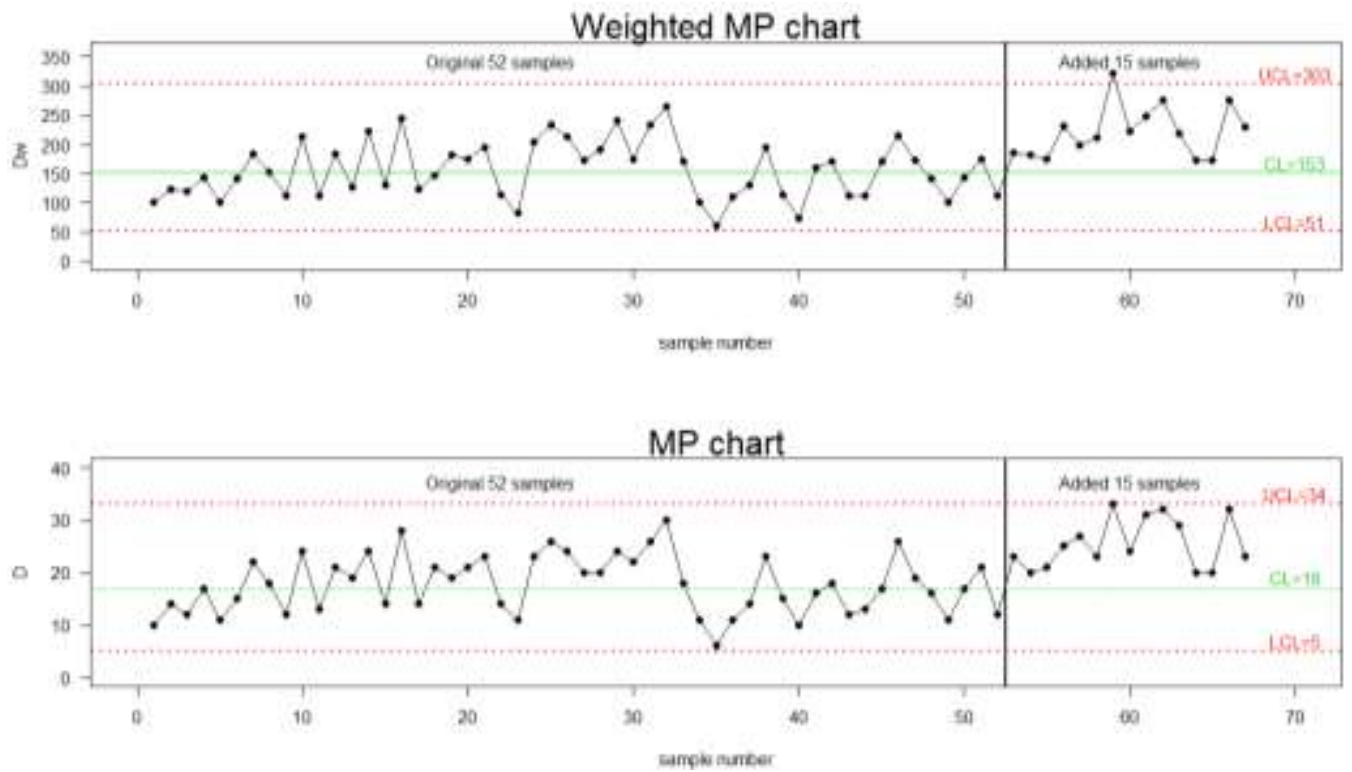


\section{Conclusions and Future Research Areas}

Considering the correlation among different quality characteristics and their degrees of influence on the final product, we propose a new statistic for demerit scheme which gives different weights to different quality characteristics according to their degrees of influence on the final product. Then, a new demerit control chart for multivariate weighted Poisson distribution (WMP chart) is developed when the impact of each quality characteristic on the final product is different. It is worthy to note that the WMP chart is designed in a general form and the MP chart proposed by Chiu and Kuo (2007) is a special case of WMP chart when the defect weights of different quality characteristics are equal to one. Moreover, a simulation study is conducted to evaluate the detecting performances of our proposed WMP chart and multivariate Poisson control chart (MP chart) using the out-of-control average run length $\left(\mathrm{ARL}_{1}\right)$. Finally, a numerical example with a two dimensional telecommunication data set is given to demonstrate the usefulness of our proposed WMP chart. It is worth to note that the determination of weights plays an important role on the detecting performance of the new demerit control chart. To assist practitioners effectively determine the weights in real application, we have also provided a rule of thumb for the preliminary hazard analysis in the numerical example section. Both the simulation results and numerical example indicate that the detecting ability of our proposed WMP chart outperforms that of the MP chart when the parameter has a shift in the quality characteristic with a serious impact on the final product for multivariate Poisson processes. Hopefully, the results of this research as well as our proposed application procedure of using the WMP chart can provide a better alternative for detecting the parameter shifts occurred in a multivariate weighted Poisson process.

Due to the time constraints, we focus on Bivariate Poisson distribution in the simulation studies. Various combinations for the different defect weights when the number of quality characteristics is equal to three or more can be further extended in performing simulation analysis. Developing a new process capability index for multivariable weighted Poisson distribution by using our proposed statistics for demerit scheme may also be considered in future research.

\section{References}

Aslam, M., Srinivasa Rao, G., Ahmad, L., and Jun, C. H. 2017. A control chart for multivariate Poisson distribution using repetitive sampling. Journal of Applied Statistics, 44(1), 123-136.

Chimka, J. R., and Cabrera Arispe, P. V. 2006. New demerit control limits for poisson distributed defects. Quality Engineering, 18(4), 461-467.

Chiu, J. E., and Kuo, T. I. 2007. Attribute control chart for multivariate Poisson distribution. Communications in Statistics-Theory and Methods, 37(1), 146-158.

Cozzucoli, P. C., and Marozzi, M. 2018. Monitoring multivariate Poisson processes: a review and some new results. Quality Technology and Quantitative Management, $15(1), 53-68$. 
Dodge, H. F. 1928. A method of rating manufactured product. Bell Labs Technical Journal, 7(2), 350-368.

He, S., He, Z., and Wang, G. A. 2014. CUSUM control charts for multivariate Poisson distribution. Communications in Statistics-Theory and Methods, 43(6), 1192-1208.

Henley, E. J., and Kumamoto, H. 1981. Reliability engineering and risk assessment (Vol. 568). Englewood Cliffs (NJ): Prentice-Hall.

Holgate, P. 1964. Estimation for the bivariate Poisson distribution. Biometrika, 51(1-2), 241-287.

Jiang, W., Au, S. T., Tsui, K. L., and Xie, M. 2002. Process monitoring with univariate and multivariate c-charts. Technical Report, the Logistics Institute, Georgia Tech, and the Logistics Institute-Asia Pacific.

Johnson, N. L. 1997. Discrete multivariate distributions (No. 04; QA273. 6, J645.).

Jones, L. A., Woodall, W. H., and Conerly, M. D. 1999. Exact properties of demerit control charts. Journal of Quality Technology, 31(2), 207-216.

Karlis, D. 2003. An EM algorithm for multivariate Poisson distribution and related models. Journal of Applied Statistics, 30(1), 63-77.

Kawamura, K. 1973. The structure of bivariate Poisson distribution. In Kodai Mathematical Seminar Reports (Vol. 25, No. 2, pp. 246-256). Department of Mathematics, Tokyo Institute of Technology.

Kawamura, K. 1979. The structure of multivariate Poisson distribution. Kodai Mathematical Journal, 2(3), 337-345.

Kawamura, K. 1984. Direct calculation of maximum likelihood estimator for the bivariate Poisson distribution. Kodai mathematical journal, 7(2), 211-221.

Kemp, C. D., and Kemp, A. W. 1965. Some properties of the 'Hermite' distribution. Biometrika, 52(3-4), 381-394.

Krishnamoorthy, A. S. 1951. Multivariate binomial and Poisson distributions. Sankhyā: The Indian Journal of Statistics, 117-124.

Laungrungrong, B., Borror, C. M., and Montgomery, D. C. 2014. A one-sided MEWMA control chart for Poisson-distributed data. International Journal of Data Analysis Techniques and Strategies, 6(1), 15-42.

Montgomery, D. C. 2009. Introduction to statistical quality control. John Wiley and Sons (New York). 